\title{
EFFECT OF Age OF A SORGHUM-SUdANGRASS HYBRID ON ITS Allelopathic ACtion
}

\author{
Efeito da Idade de um Híbrido de Sorgo com Capim-Sudão em sua Ação Alelopática
}

\author{
MARCHI, G. ${ }^{2}$, MARCHI, E.C.S. ${ }^{3}$, WANG, G. ${ }^{4}$ and MCGIFFEN, M. ${ }^{5}$
}

\begin{abstract}
Hybrids of Sorghum sudanensis (sudangrass) and Sorghum bicolor genotypes can produce high amounts of biomass, sorgoleone (a long chain hydroquinone), and other phytotoxic substances. Shoots and roots of a sorghum-sudangrass hybrid (cv. Trudan 8) were collected 10, 20,30,40, and 50 days after emergence. Four concentrations of aqueous extracts from the shoots and roots $\left(0,0.4,2\right.$, and $\left.10 \mathrm{~g} \mathrm{~L}^{-1}, w / v\right)$ were used to treat seeds of lettuce (Lactuca sativa), tomato (Lycopersicum sculentum), purslane (Portulaca oleracea), and pigweed (Amaranthus retroflexus). Seed germination of lettuce, tomato, and pigweed was inhibited by extracts from sorghum-sudangrass shoots at $10 \mathrm{~g} \mathrm{~L}^{-1}$ when made from sorghum-sudangrass plants 20 days or less in age. Seed germination of purslane was not inhibited by any sorghum-sudangrass extract. Growth of the four species evaluated were systematically inhibited when treated with 10 $\mathrm{g} \mathrm{L}^{-1}$ extracts from sorghum-sudangrass shoots harvested up to 10 days after emergence.
\end{abstract}

Keywords: allelopathy, germination inhibition, lettuce (Lactuca sativa), tomato (Lycopersicum sculentum), pigweed (Amaranthus retroflexus) and purslane (Portulaca oleracea).

RESUMO - Os capins híbridos obtidos pelo cruzamento entre Sorghum sudanensis (capimsudão) e genótipos de Sorghum bicolor possuem alto potencial para produção de biomassa e para controle de plantas daninhas pela produção de substâncias fitotóxicas, como o sorgoleone (uma hidroquinona de cadeia longa). Sementes de alface (Lactuca sativa), tomate (Lycopersicum sculentum), beldroega (Portulaca oleracea) e caruru (Amaranthus retroflexus) foram submetidas a tratamentos com extratos aquosos da parte aérea e das raízes do híbrido de sorgo com capim-sudão, cv. Trudan 8, colhido em cinco diferentes estádios de crescimento (10, 20, 30, 40 e 50 dias após a emergência). Os extratos foram preparados em quatro concentrações $\left(0,0,4,2\right.$ e $\left.10 \mathrm{~g} \mathrm{~L}^{-1}, \mathrm{p} / \mathrm{v}\right)$ e aplicados em quatro repetições. Após os tratamentos, a germinação e o comprimento de plântulas das espécies foram avaliados. A germinação de sementes de tomate, caruru e alface foi inibida pelos extratos da parte aérea das plantas de Trudan 8, na concentração de $10 \mathrm{~g} \mathrm{~L}{ }^{1}$, colhidas até os 20 dias após a emergência. A germinação de sementes de beldroega, no tocante à porcentagem de germinação, não foi inibida pelos extratos de Trudan 8. O crescimento das quatro espécies avaliadas foi inibido quando tratadas com extratos aquosos da parte aérea de Trudan 8, colhida até os 10 dias após a emergência, na concentração de $10 \mathrm{~g} \mathrm{~L}^{-1}$.

Palavras-chave: alelopatia, inibidor de germinação, alface (Lactuca sativa), tomate (Lycopersicum sculentum), caruru (Amaranthus retroflexus) e beldroega (Portulaca oleracea).

1 Recebido para publicação em 19.5.2008 e na forma revisada em 19.9.2008.

2 Pesquisador, Embrapa Cerrados; Rodovia BR020, km 18, Caixa Postal 08223, 73310-970, Planaltina-DF, <giuliano.marchi@cpac.embrapa.br>; ${ }^{3}$ Bolsista, CNPq/Embrapa Cerrados; ${ }^{4}$ Pesquisador, Maricopa Agricultural Center, University of Arizona, 37860 W. Smith-Enke Road, Maricopa, AZ 85239, USA; ${ }^{5}$ Prof., Department of Botany and Plant Sciences, University of California, Riverside, CA 92521, USA

Planta Daninha, Viçosa-MG, v. 26, n. 4, p. 707-716, 2008 


\section{INTRODUCTION}

Both organic and conventional farmers apply no tillage systems. Among the crops used for pasture and straw mulch for integrated crop-livestock production, cutting or foraging sorghum-sudangrass hybrids produce large amounts of biomass, suppress weeds, and decrease soil compaction (McKinney et al., 2004). Sorghum-sudangrass cultivars are produced from crossing inter-specific Sorghum sudanensis Piper Stapf hybrids with Sorghum bicolor genotypes (Raupp \& Brancão, 2000). Growing these hybrids without irrigation during February in Central Brazil has yielded 9 to 12 tons of dry matter in three successive cuts, for a total of at least 40 to 60 tons of fresh forage per hectare (Zago, 1997; Mello et al., 2003). Sorghum-sudangrass hybrids also present high forage production potential when grown at the beginning of the rainy season in Central Brazil (Tomich et al., 2004).

Besides its high biomass production potential, sorghum-sudangrass hybrids release phytotoxic substances into the environment, being able to suppress weed development in some situations (Forney \& Foy, 1985). Sorghum species produce a myriad of substances with allelopathic potential that may inhibit weed germination and growth (Weston, 1996). The allelopathic compounds are a secondary metabolism product, and their chemical compositions and concentrations vary according to species, age, and climatic conditions (Dayan, 2006). Using allelopathic substance-producing plants may allow farmers to reduce herbicide use (Weston, 1996; Bàrberi \& Mazzoncini, 2001).

Allelopathic effects of sorghum-sudangrass may be related to phenolic compounds released by tissue decomposition (Weston et al., 1989; Sene et al., 2001). Phenolic acids such as ferulic, $p$-coumaric, syringic, vanilic, and $p$ hydroxybenzoic are produced from sorghum hybrid roots during residue decomposition. These substances are responsible for growth inhibition of several plant species (Roth et al., 2000). Another compound found in sorghum genotypes is dhurrin, a cianogenic glycoside that degrades to $p$ hydroxybenzaldeide, $\mathrm{HCN}$, and glucose (Nicollier et al., 1983).

Netzley \& Butler (1986) verified that sorghum roots exude the long chain hydroquinone sorgoleone, which exhibits high phytotoxicity. This substance is a potent electron transport inhibitor in photosystem II in chloroplasts (González et al., 1997; Hej1 \& Koster, 2004). Sudangrass also produces from 1.3 to $1.9 \mathrm{mg}$ sorgoleone $\mathrm{g}^{-1}$, an amount similar to that produced by sorghum (Bais et al., 2006). Sorgoleone is active at very small concentrations (McGuire, 2003) and has a higher specific activity than diuron, one of the most potent photosynthetic inhibitors (Gonzalez et al., 1997).

Sorgoleone is rapidly degraded in soil (Czarnota et al., 2001), suggesting that large amounts must be continuously produced to maintain phytotoxicity in the soil solution (Bais et al., 2006). However, the capacity to produce plant allelochemicals declines as the plant ages (Woodhead, 1981; Weston et al., 1989; Ben-Hammouda et al., 1995; An et al., 2003), thus sorgoleone is mainly produced in younger plants (Dayan, 2006).

The aim of this work was to evaluate inhibition of lettuce, tomato, purslane, and pigweed seed germination and growth after treatment with shoot and root aqueous extracts of the sorghum-sudangrass hybrid cv. Trudan 8 harvested on five dates after germination.

\section{MATERIALS AND METHODS}

\section{Extraction procedure}

Seeds of sorghum (Sorghum bicolor) and sudangrass (Sorghum sudanensis) hybrid, cv. Trudan 8 (hereafter Sorghum-sudangrass), were planted in 32 pots under glasshouse conditions at the University of California, Riverside from August to November 2003. Average daily temperature during the experiment varied from 13 to $28^{\circ} \mathrm{C}$.

Plants were harvested at five ages: 10, 20, 30,40 , and 50 days after emergence. After each harvest, plants were washed with distilled water, separated as shoots and roots, and dried in oven at $60{ }^{\circ} \mathrm{C}$. Shoots and roots were ground to a fine powder and extracted with distilled water for $24 \mathrm{~h}$ at $24^{\circ} \mathrm{C}$ in a lit room. These extracts were filtered through four tissue sheets to remove particles and 
filtered again with Whatman n.42. Four concentrations $\left(0,0.4,2\right.$, and $\left.10 \mathrm{~g} \mathrm{~L}^{-1} \mathrm{w} / \mathrm{v}\right)$ of each extract were prepared and stored in the dark for 7 days at $4^{\circ} \mathrm{C}$ for further analysis. Electrical conductivity of the extracts ranged from $35 \mu \mathrm{S} / \mathrm{cm}$ to $1.51 \mathrm{mS} / \mathrm{cm}$ in the shoot extracts and from $15 \mu \mathrm{S} / \mathrm{cm}$ to $1.32 \mathrm{mS} / \mathrm{cm}$ in the root extracts, while $\mathrm{pH}$ ranged from 5.64 to 6.59 in the shoots and from 4.11 to 6.77 in the roots. These values were within the normal range and did not harm the germination or development of the plants (Everitt et al., 1983).

\section{Seed germination and plant growth}

Germination of lettuce (Lactuca sativa) cv. "Black Seed Simpson", tomato (Lycopersicum sculentum) cv. "Advantage 81376", purslane (Portulaca oleracea), and pigweed (Amaranthus retroflexus) seeds was measured after treatment with sorghum-sudangrass root and shoot extracts. The experiment was arranged in a completely randomized design with four replicates. For each replicate, thirty seeds of each species were placed into two petri dishes lined with sterile filter paper (Whatman \#1). Seven $\mathrm{mL}$ of distilled water (control) or one of the four sorghum-sudangrass extracts were added to each Petri dish, totaling five experimental treatments. Petri dishes were sealed to prevent humidity loss or contamination, and kept in a lit room for $10 \mathrm{~h}$ followed by $14 \mathrm{~h}$ in the dark at $24^{\circ} \mathrm{C}$. Lettuce, tomato, purslane, and pigweed seed germination and plant growth were measured $4,5,6$, and 7 days after sowing, respectively. Seeds were counted as germinated after radicle emergence of at least $1 \mathrm{~mm}$. Seven plants were randomly chosen from each Petri dish and their size measured.

\section{Data analysis}

Germination data were submitted to analysis at $5 \%$ probability confidence intervals. The data were binomially distributed and analyzed by calculating exact confidence intervals (Pratt, 1968; Blyth, 1996; Leemis \& Trivedi, 1996) using Sisvar software (Ferreira, 2000). The statistical model used was $\mathrm{Y} / \mathrm{n}$ Binomial (", n), where: Y denotes the number of successes (germinated seeds), $\mathrm{n}$ denotes the number of observations (total seeds), and ", the proportion of population successes for each sample.

Plant growth data were subjected to analysis of variance and the means were separated by the Tukey's test (5\% probability).

\section{RESULTS AND DISCUSSION}

\section{Seed germination}

Seed germination of lettuce, tomato, and pigweed was inhibited by extracts from Sorghum-sudangrass plants harvested at 10 and 20 days after emergence, at $10 \mathrm{~g} \mathrm{~L}^{-1}$ (Table 1). Root and shoot extracts prepared with Sorghum-sudangrass plants harvested 30, 40, and 50 days after emergence did not inhibit germination of these three species, suggesting that the allelopathic potential of sorghum-sudangrass plants harvested 30 days after germination decreased.

Lettuce seed germination was inhibited when using 2 and $10 \mathrm{~g} \mathrm{~L}^{-1}$ sorghum-sudangrass shoot and root extracts from plants harvested 10 days after emergence. Extracts of sorghumsudangrass prepared with plants harvested 20 to 50 days after emergence did not inhibit lettuce seed germination, except $10 \mathrm{~g} \mathrm{~L}^{-1}$ extracts of sorghum-sudangrass shoot harvested 20 days after emergence, which did inhibit lettuce seed germination. Tomato and pigweed seed germination percentage was strongly reduced following application of $10 \mathrm{~g} \mathrm{~L}^{-1}$ extracts of sorghum-sudangrass shoot harvested 10 or 20 days after emergence. Purslane seed germination was not inhibited by extracts of sorghum-sudangrass shoot or root.

\section{Plant growth}

As sorgoleone produced by sorghumsudangrass affects photosystem II (González et al., 1997; Hejl et al., 2004), it is expected that the growth of other plants be affected intensely just after they germinate and start to photosynthesize (Barbosa et al., 1998). The effects of sorghum-sudangrass extracts on plant growth depended on the type of extract: root or shoot, its concentration, and its age at 
Table 1 - Lettuce, tomato, purslane, and pigweed germination (\%) after treatment with root or shoot aqueous extracts of sorghumsudangrass harvested at 5 growth stages ${ }^{\S}$

\begin{tabular}{|c|c|c|c|c|c|}
\hline \multirow{3}{*}{$\begin{array}{c}\text { Extract } \\
\text { concentration } \\
\left(\mathrm{g} \mathrm{L}^{-1}\right)\end{array}$} & \multicolumn{5}{|c|}{ Sorghum-sudangrass age (days after emergence) } \\
\hline & 10 & 20 & 30 & 40 & 50 \\
\hline & \multicolumn{5}{|c|}{ Germination (\%) } \\
\hline & \multicolumn{5}{|c|}{ Lettuce } \\
\hline 0 & $95.00 \mathrm{Aa}$ & $95.00 \mathrm{Aa}$ & $94.58 \mathrm{Aa}$ & $95.83 \mathrm{Aa}$ & $94.17 \mathrm{Aa}$ \\
\hline $0.4 \mathrm{R}^{*}$ & $95.42 \mathrm{Aa}$ & $94.17 \mathrm{Aa}$ & $87.08 \mathrm{Aab}$ & $95.83 \mathrm{Aa}$ & $95.83 \mathrm{Aa}$ \\
\hline $2 \mathrm{R}$ & 83.33 ABab & $94.58 \mathrm{Aa}$ & $87.08 \mathrm{Aa}$ & $93.33 \mathrm{Aa}$ & $92.50 \mathrm{Aa}$ \\
\hline $10 \mathrm{R}$ & $82.92 \mathrm{ABab}$ & $92.08 \mathrm{Aa}$ & $83.75 \mathrm{ABab}$ & $95.00 \mathrm{Aa}$ & $95.83 \mathrm{Aa}$ \\
\hline $0.4 \mathrm{~S}$ & $91.25 \mathrm{Aa}$ & $94.17 \mathrm{Aa}$ & 87.08 Aab & $95.83 \mathrm{Aa}$ & $96.67 \mathrm{Aa}$ \\
\hline $2 \mathrm{~S}$ & $82.92 \mathrm{ABab}$ & $95.00 \mathrm{Aa}$ & $88.75 \mathrm{Aa}$ & $93.33 \mathrm{Aa}$ & $95.83 \mathrm{Aa}$ \\
\hline \multirow[t]{2}{*}{$10 \mathrm{~S}$} & $64.58 \mathrm{Cb}$ & $84.58 \mathrm{ABa}$ & $89.58 \mathrm{Aa}$ & $92.50 \mathrm{Aa}$ & $91.67 \mathrm{Aa}$ \\
\hline & \multicolumn{5}{|c|}{ Tomato } \\
\hline 0 & $66.67 \mathrm{Aa}$ & $76.25 \mathrm{Aa}$ & $72.50 \mathrm{Aa}$ & $70.00 \mathrm{Aa}$ & $66.67 \mathrm{Aa}$ \\
\hline $0.4 \mathrm{R}$ & $70.41 \mathrm{Aa}$ & $69.17 \mathrm{Aa}$ & $68.75 \mathrm{Aa}$ & $75.83 \mathrm{Aa}$ & $67.50 \mathrm{Aa}$ \\
\hline $2 \mathrm{R}$ & $75.00 \mathrm{Aa}$ & $71.67 \mathrm{Aa}$ & $66.67 \mathrm{Aa}$ & $73.33 \mathrm{Aa}$ & $72.50 \mathrm{Aa}$ \\
\hline $10 \mathrm{R}$ & $70.00 \mathrm{Aa}$ & $68.10 \mathrm{Aa}$ & $71.66 \mathrm{Aa}$ & $68.33 \mathrm{Aa}$ & $71.67 \mathrm{Aa}$ \\
\hline $0.4 \mathrm{~S}$ & $65.00 \mathrm{Aa}$ & $71.25 \mathrm{Aa}$ & $65.42 \mathrm{Aa}$ & $75.83 \mathrm{Aa}$ & $67.50 \mathrm{Aa}$ \\
\hline $2 \mathrm{~S}$ & $60.83 \mathrm{Aa}$ & $73.75 \mathrm{Aa}$ & $68.33 \mathrm{Aa}$ & $69.17 \mathrm{Aa}$ & $68.33 \mathrm{Aa}$ \\
\hline \multirow[t]{2}{*}{$10 \mathrm{~S}$} & $12.08 \mathrm{Bb}$ & $3.75 \mathrm{Bc}$ & $65.00 \mathrm{Aa}$ & $71.67 \mathrm{Aa}$ & $66.67 \mathrm{Aa}$ \\
\hline & \multicolumn{5}{|c|}{ Purslane } \\
\hline 0 & $81.25 \mathrm{Aa}$ & $75.00 \mathrm{Aa}$ & $78.75 \mathrm{Aa}$ & $72.50 \mathrm{Aa}$ & $78.33 \mathrm{Aa}$ \\
\hline $0.4 \mathrm{R}$ & $79.17 \mathrm{Aa}$ & $80.83 \mathrm{Aa}$ & $78.33 \mathrm{ABa}$ & $82.50 \mathrm{Aa}$ & $76.67 \mathrm{Aa}$ \\
\hline $2 \mathrm{R}$ & $81.67 \mathrm{Aa}$ & $81.67 \mathrm{Aa}$ & $80.83 \mathrm{Aa}$ & $69.17 \mathrm{ABb}$ & $82.50 \mathrm{Aa}$ \\
\hline $10 \mathrm{R}$ & 79.17 Aa & $80.83 \mathrm{Aa}$ & $78.33 \mathrm{ABa}$ & $80.00 \mathrm{Aa}$ & $77.50 \mathrm{Aa}$ \\
\hline $0.4 \mathrm{~S}$ & $79.17 \mathrm{Aa}$ & $74.17 \mathrm{Aa}$ & $80.83 \mathrm{Aa}$ & $75.83 \mathrm{Aa}$ & $76.67 \mathrm{Aa}$ \\
\hline $2 \mathrm{~S}$ & $80.00 \mathrm{Aa}$ & $74.58 \mathrm{Aab}$ & 89.17 Aa & $78.33 \mathrm{Aab}$ & $80.83 \mathrm{Aa}$ \\
\hline \multirow[t]{2}{*}{$10 \mathrm{~S}$} & $76.25 \mathrm{Aa}$ & $75.42 \mathrm{Aa}$ & $74.17 \mathrm{ABa}$ & $73.33 \mathrm{Aa}$ & $84.17 \mathrm{Aa}$ \\
\hline & \multicolumn{5}{|c|}{ Pigweed } \\
\hline 0 & $90.00 \mathrm{Aa}$ & $91.67 \mathrm{Aa}$ & $92.08 \mathrm{Aa}$ & $90.00 \mathrm{ABa}$ & $96.67 \mathrm{Aa}$ \\
\hline $0.4 \mathrm{R}$ & $89.58 \mathrm{Aa}$ & $88.33 \mathrm{Aa}$ & $90.42 \mathrm{Aa}$ & $91.67 \mathrm{ABa}$ & $89.17 \mathrm{ABa}$ \\
\hline $2 \mathrm{R}$ & $87.50 \mathrm{Aa}$ & $86.25 \mathrm{Aa}$ & 83.33 Aab & $94.17 \mathrm{Aa}$ & $88.33 \mathrm{ABa}$ \\
\hline $10 \mathrm{R}$ & $89.05 \mathrm{Ab}$ & $87.50 \mathrm{Ab}$ & $88.33 \mathrm{Ab}$ & $98.33 \mathrm{Aa}$ & $90.00 \mathrm{Aa}$ \\
\hline $0.4 \mathrm{~S}$ & $80.42 \mathrm{Aab}$ & 86.67 Aab & $89.58 \mathrm{Aa}$ & $96.67 \mathrm{Aa}$ & $93.33 \mathrm{Aa}$ \\
\hline $2 \mathrm{~S}$ & $82.08 \mathrm{Aab}$ & $85.42 \mathrm{Aab}$ & $89.58 \mathrm{Aab}$ & $97.50 \mathrm{Aa}$ & $95.83 \mathrm{Aa}$ \\
\hline $10 \mathrm{~S}$ & $2.92 \mathrm{Bb}$ & $2.08 \mathrm{Bb}$ & $85.83 \mathrm{Aa}$ & $95.00 \mathrm{Aa}$ & $90.00 \mathrm{Aa}$ \\
\hline
\end{tabular}

$\S$ Different concentrations $\left(0.4,2,10 \mathrm{~g} \mathrm{~L}^{-1}\right)$ of root $(\mathrm{R})$ or shoot $(\mathrm{S})$ extracts from sorghum-sudangrass plants harvested at 5 different stages $(10,20,30,40,50$ days after germination) were used to treat the seeds of four plant species (lettuce, tomato, purslane, and pigweed). For each species, plant length in the same column followed by the same upper case letter was not significantly different, while values in the same rows followed by the same lower case letter were not significantly different by the Tukey test $\mathrm{p} \leq 5 \%$.

$* \mathrm{R}=$ root extracts; $\mathrm{S}=$ shoot extracts. 
harvest (Table 2). Shoot extracts inhibited growth more strongly than those prepared from roots. This result is consistent with Correia et al. (2005a), who showed that leaf extracts from two sorghum genotypes, XBG00478 and DKB860, inhibited soybean radicle length more than the root extracts.

Extracts of $10 \mathrm{~g} \mathrm{~L}^{-1}$ sorghum-sudangrass prepared from shoot harvested at 10 and 20 days after emergence strongly inhibited the growth of all the plant species (Table 2). Sorghum-sudangrass 2 and $10 \mathrm{~g} \mathrm{~L}^{-1}$ extracts prepared from shoot or root harvested 10 days after emergence, inhibited lettuce, tomato, purslane, and pigweed plant length. However, $10 \mathrm{~g} \mathrm{~L}^{-1}$ extracts prepared with shoots strongly inhibited seedling growth, mainly when shoot was harvested 10 and 20 days after emergence. Tomato and pigweed seedlings either grew poorly or did not grow at all when treated with $10 \mathrm{~g} \mathrm{~L} \mathrm{~L}^{-1}$ extracts prepared from shoots harvested 10 and 20 days after emergence.

Correia et al. (2005a) suggest that radicle inhibition by allelopathic substances in Petri dish experiments is more likely to occur than shoot inhibition, as absorption and concentration of allelochemicals by root tissues are favored by physical contact with the filter paper. In a field experiment, Xuan et al. (2004) determined that plant leaves produce more allelochemicals and thus have higher allelopathic potential than roots or stems. We found that both shoots and roots were strongly inhibited by sorghum-sudangrass extracts (Tables 3 and 4).

Lettuce, tomato, purslane, and pigweed radicle and shoot growth was strongly inhibited following treatment with 0.4, 2 and $10 \mathrm{~g} \mathrm{~L}^{-1}$ sorghum-sudangrass shoot extracts, especially when the extracts were prepared with sorghum-sudangrass harvested 10 days after emergence (Table 3). Any of the $10 \mathrm{~g} \mathrm{~L}^{-1}$ sorghum-sudangrass shoot extracts other than those prepared from 30-day-old plants inhibited radicle growth of all the target species. Tomato and pigweed roots and shoots either grew minimally or not at all when treated with
$10 \mathrm{~g} \mathrm{~L}^{-1}$ extracts prepared with shoots harvested 10 and 20 days after emergence.

Sorghum-sudangrass extracts inhibited both seed germination and growth. Ten $\mathrm{g} \mathrm{L}^{-1}$ sorghum-sudangrass extracts inhibited lettuce, tomato, purslane and pigweed germination and root and shoot growth, mainly when sorghum-sudangrass shoot extracts were prepared from 10-day-old plants. This result is important mainly when sorghumsudangrass is planted with other crops in croplivestock integration and affects the productions of other crops (Correia et al., 2005b; Olibone et al., 2006).

Since older sorghum-sudangrass tissue presents a lower concentration of allelopathic chemicals, high biomass production does not always mean high allelopathic potential. These results suggest that sorghumsudangrass allelochemicals are produced at a higher intensity up to 10 days after its emergence, and are found primarily in the shoots. The allelopathic potential of aqueous extracts from shoots of the hybrid Sudex [(Sorghum bicolor) Moench x Sorghum Sudanese (Piper) Stapf), cv. FFR 201] peaks 7 days after germination (Weston et al., 1989). In the present experiment, extracts prepared with sorghum-sudangrass harvested at 30,40, and 50 days after emergence did not inhibit seed germination (Table 1) and seedling growth (Table 2). These results corroborate the hypothesis that with age, the allelopathic potential of sorghum and its hybrids decreases.

Lettuce, tomato, and pigweed seed germination and growth inhibition by sorghum-sudangrass extracts suggest that this hybrid may have the potential to model plant communities and serve as an important alien species when introduced in fragile natural ecosystems such as Cerrado (Bais et al., 2006). When introduced into native ecosystems, sorghum-sudangrass may play a role as an alien species with high biomass production potential and allelochemical production able to suppresses the growth of other species (Trezzi \& Vidal, 2004; Vidal \& Trezzi, 2004). 
Table 2 - Lettuce, tomato, purslane, and pigweed plant length after being treated with aqueous extracts of sorghum-sudangrass root and shoot harvested at 5 growth stages $\S$

\begin{tabular}{|c|c|c|c|c|c|}
\hline \multirow{3}{*}{$\begin{array}{c}\text { Extract } \\
\text { concentration } \\
\left(\mathrm{g} \mathrm{L}^{-1}\right)\end{array}$} & \multicolumn{5}{|c|}{ Sorghum-sudangrass age (days after emergence) } \\
\hline & 10 & 20 & 30 & 40 & 50 \\
\hline & \multicolumn{5}{|c|}{ Germination (\%) } \\
\hline & \multicolumn{5}{|c|}{ Lettuce } \\
\hline 0 & $95.00 \mathrm{Aa}$ & $95.00 \mathrm{Aa}$ & $94.58 \mathrm{Aa}$ & $95.83 \mathrm{Aa}$ & $94.17 \mathrm{Aa}$ \\
\hline $0.4 \mathrm{R}^{*}$ & $95.42 \mathrm{Aa}$ & $94.17 \mathrm{Aa}$ & $87.08 \mathrm{Aab}$ & $95.83 \mathrm{Aa}$ & $95.83 \mathrm{Aa}$ \\
\hline $2 \mathrm{R}$ & $83.33 \mathrm{ABab}$ & $94.58 \mathrm{Aa}$ & $87.08 \mathrm{Aa}$ & $93.33 \mathrm{Aa}$ & $92.50 \mathrm{Aa}$ \\
\hline $10 \mathrm{R}$ & $82.92 \mathrm{ABab}$ & $92.08 \mathrm{Aa}$ & $83.75 \mathrm{ABab}$ & $95.00 \mathrm{Aa}$ & $95.83 \mathrm{Aa}$ \\
\hline $0.4 \mathrm{~S}$ & $91.25 \mathrm{Aa}$ & $94.17 \mathrm{Aa}$ & $87.08 \mathrm{Aab}$ & $95.83 \mathrm{Aa}$ & $96.67 \mathrm{Aa}$ \\
\hline $2 \mathrm{~S}$ & $82.92 \mathrm{ABab}$ & $95.00 \mathrm{Aa}$ & $88.75 \mathrm{Aa}$ & $93.33 \mathrm{Aa}$ & $95.83 \mathrm{Aa}$ \\
\hline \multirow[t]{2}{*}{$10 \mathrm{~S}$} & $64.58 \mathrm{Cb}$ & $84.58 \mathrm{ABa}$ & $89.58 \mathrm{Aa}$ & $92.50 \mathrm{Aa}$ & $91.67 \mathrm{Aa}$ \\
\hline & \multicolumn{5}{|c|}{ Tomato } \\
\hline 0 & $66.67 \mathrm{Aa}$ & $76.25 \mathrm{Aa}$ & $72.50 \mathrm{Aa}$ & $70.00 \mathrm{Aa}$ & $66.67 \mathrm{Aa}$ \\
\hline $0.4 \mathrm{R}$ & $70.41 \mathrm{Aa}$ & $69.17 \mathrm{Aa}$ & $68.75 \mathrm{Aa}$ & $75.83 \mathrm{Aa}$ & $67.50 \mathrm{Aa}$ \\
\hline $2 \mathrm{R}$ & $75.00 \mathrm{Aa}$ & $71.67 \mathrm{Aa}$ & $66.67 \mathrm{Aa}$ & $73.33 \mathrm{Aa}$ & $72.50 \mathrm{Aa}$ \\
\hline $10 \mathrm{R}$ & $70.00 \mathrm{Aa}$ & $68.10 \mathrm{Aa}$ & $71.66 \mathrm{Aa}$ & $68.33 \mathrm{Aa}$ & $71.67 \mathrm{Aa}$ \\
\hline $0.4 \mathrm{~S}$ & $65.00 \mathrm{Aa}$ & $71.25 \mathrm{Aa}$ & $65.42 \mathrm{Aa}$ & $75.83 \mathrm{Aa}$ & $67.50 \mathrm{Aa}$ \\
\hline $2 \mathrm{~S}$ & $60.83 \mathrm{Aa}$ & $73.75 \mathrm{Aa}$ & $68.33 \mathrm{Aa}$ & $69.17 \mathrm{Aa}$ & $68.33 \mathrm{Aa}$ \\
\hline \multirow[t]{2}{*}{$10 \mathrm{~S}$} & $12.08 \mathrm{Bb}$ & $3.75 \mathrm{Bc}$ & $65.00 \mathrm{Aa}$ & $71.67 \mathrm{Aa}$ & $66.67 \mathrm{Aa}$ \\
\hline & \multicolumn{5}{|c|}{ Purslane } \\
\hline 0 & $81.25 \mathrm{Aa}$ & $75.00 \mathrm{Aa}$ & $78.75 \mathrm{Aa}$ & $72.50 \mathrm{Aa}$ & $78.33 \mathrm{Aa}$ \\
\hline $0.4 \mathrm{R}$ & 79.17 Aa & $80.83 \mathrm{Aa}$ & $78.33 \mathrm{ABa}$ & $82.50 \mathrm{Aa}$ & $76.67 \mathrm{Aa}$ \\
\hline $2 \mathrm{R}$ & $81.67 \mathrm{Aa}$ & $81.67 \mathrm{Aa}$ & $80.83 \mathrm{Aa}$ & $69.17 \mathrm{ABb}$ & $82.50 \mathrm{Aa}$ \\
\hline $10 \mathrm{R}$ & 79.17 Aa & $80.83 \mathrm{Aa}$ & $78.33 \mathrm{ABa}$ & $80.00 \mathrm{Aa}$ & $77.50 \mathrm{Aa}$ \\
\hline $0.4 \mathrm{~S}$ & $79.17 \mathrm{Aa}$ & $74.17 \mathrm{Aa}$ & $80.83 \mathrm{Aa}$ & $75.83 \mathrm{Aa}$ & $76.67 \mathrm{Aa}$ \\
\hline $2 \mathrm{~S}$ & $80.00 \mathrm{Aa}$ & 74.58 Aab & $89.17 \mathrm{Aa}$ & $78.33 \mathrm{Aab}$ & $80.83 \mathrm{Aa}$ \\
\hline \multirow[t]{2}{*}{$10 \mathrm{~S}$} & $76.25 \mathrm{Aa}$ & $75.42 \mathrm{Aa}$ & $74.17 \mathrm{ABa}$ & $73.33 \mathrm{Aa}$ & $84.17 \mathrm{Aa}$ \\
\hline & \multicolumn{5}{|c|}{ Pigweed } \\
\hline 0 & $90.00 \mathrm{Aa}$ & $91.67 \mathrm{Aa}$ & $92.08 \mathrm{Aa}$ & $90.00 \mathrm{ABa}$ & $96.67 \mathrm{Aa}$ \\
\hline $0.4 \mathrm{R}$ & $89.58 \mathrm{Aa}$ & $88.33 \mathrm{Aa}$ & $90.42 \mathrm{Aa}$ & $91.67 \mathrm{ABa}$ & $89.17 \mathrm{ABa}$ \\
\hline $2 \mathrm{R}$ & $87.50 \mathrm{Aa}$ & $86.25 \mathrm{Aa}$ & 83.33 Aab & $94.17 \mathrm{Aa}$ & $88.33 \mathrm{ABa}$ \\
\hline $10 \mathrm{R}$ & $89.05 \mathrm{Ab}$ & $87.50 \mathrm{Ab}$ & $88.33 \mathrm{Ab}$ & $98.33 \mathrm{Aa}$ & $90.00 \mathrm{Aa}$ \\
\hline $0.4 \mathrm{~S}$ & $80.42 \mathrm{Aab}$ & 86.67 Aab & $89.58 \mathrm{Aa}$ & $96.67 \mathrm{Aa}$ & $93.33 \mathrm{Aa}$ \\
\hline $2 \mathrm{~S}$ & $82.08 \mathrm{Aab}$ & $85.42 \mathrm{Aab}$ & 89.58 Aab & $97.50 \mathrm{Aa}$ & $95.83 \mathrm{Aa}$ \\
\hline $10 \mathrm{~S}$ & $2.92 \mathrm{Bb}$ & $2.08 \mathrm{Bb}$ & $85.83 \mathrm{Aa}$ & $95.00 \mathrm{Aa}$ & $90.00 \mathrm{Aa}$ \\
\hline
\end{tabular}

$\S$ Different concentrations $\left(0.4,2,10 \mathrm{~g} \mathrm{~L}^{-1}\right)$ of root $(\mathrm{R})$ or shoot $(\mathrm{S})$ extracts from sorghum-sudangrass plants harvested at 5 different stages $(10,20,30,40,50$ days after germination) were used to treat the seeds of four plant species (lettuce, tomato, purslane, and pigweed). For each species, plant length in the same column followed by the same upper case letter was not significantly different, while values in the same rows followed by the same lower case letter were not significantly different by the Tukey test pd" $5 \%$.

$* \mathrm{R}=$ root extracts; $\mathrm{S}=$ shoot extracts. 
Effect of age of a sorghum-sudangrass hybrid on its...

Table 3 - Lettuce, tomato, purslane, and pigweed root length after being treated with root and shoot aqueous extracts from sorghum-sudangrass harvested at 5 growth stages ${ }^{\S}$

\begin{tabular}{|c|c|c|c|c|c|}
\hline \multirow{3}{*}{$\begin{array}{l}\text { Extract concentration } \\
\qquad\left(\mathrm{g} \mathrm{L}^{-1}\right)\end{array}$} & \multicolumn{5}{|c|}{ Sorghum-sudangrass age (days after emergence) } \\
\hline & 10 & 20 & 30 & 40 & 50 \\
\hline & \multicolumn{5}{|c|}{ Radicle (mm) } \\
\hline & \multicolumn{5}{|c|}{ Lettuce } \\
\hline 0 & $18.01 \mathrm{Ab}$ & $13.53 \mathrm{Ac}$ & $13.39 \mathrm{Bc}$ & $19.89 \mathrm{Ab}$ & $27.67 \mathrm{Aa}$ \\
\hline $0.4 \mathrm{R}^{*}$ & $16.02 \mathrm{ABa}$ & $7.46 \mathrm{Bb}$ & $15.07 \mathrm{ABa}$ & $9.82 \mathrm{CDb}$ & $9.25 \mathrm{Cb}$ \\
\hline $2 \mathrm{R}$ & $13.04 \mathrm{BCb}$ & $12.60 \mathrm{Ab}$ & $18.49 \mathrm{Aa}$ & $17.28 \mathrm{ABa}$ & $17.57 \mathrm{Ba}$ \\
\hline $10 \mathrm{R}$ & $10.09 \mathrm{Cb}$ & $12.00 \mathrm{Aab}$ & $15.53 \mathrm{ABa}$ & $13.60 \mathrm{BCab}$ & $9.96 \mathrm{Cb}$ \\
\hline $0.4 \mathrm{~S}$ & $12.89 \mathrm{BCc}$ & $15.93 \mathrm{Abc}$ & $12.64 \mathrm{Bc}$ & $18.89 \mathrm{Ab}$ & $25.49 \mathrm{Aa}$ \\
\hline $2 \mathrm{~S}$ & $3.33 \mathrm{Dc}$ & $12.14 \mathrm{Ab}$ & 13.46 Bab & $16.42 \mathrm{ABa}$ & 15.82 Bab \\
\hline \multirow[t]{2}{*}{$10 \mathrm{~S}$} & $2.43 \mathrm{Dc}$ & $3.53 \mathrm{Bc}$ & $12.64 \mathrm{Ba}$ & $8.58 \mathrm{Db}$ & $7.82 \mathrm{Cb}$ \\
\hline & \multicolumn{5}{|c|}{ Tomato } \\
\hline 0 & $35.00 \mathrm{ABb}$ & $56.45 \mathrm{Aa}$ & $48.66 \mathrm{Aab}$ & $35.22 \mathrm{ABb}$ & $37.83 \mathrm{Ab}$ \\
\hline $0.4 \mathrm{R}$ & $34.35 \mathrm{ABb}$ & $51.75 \mathrm{Aa}$ & $48.53 \mathrm{Aa}$ & $17.67 \mathrm{BCb}$ & $26.90 \mathrm{ABb}$ \\
\hline $2 \mathrm{R}$ & $50.39 \mathrm{Aab}$ & 49.41 Aab & $60.85 \mathrm{Aa}$ & $38.71 \mathrm{Abc}$ & $24.39 \mathrm{ABc}$ \\
\hline $10 \mathrm{R}$ & $33.85 \mathrm{ABbc}$ & 49.96 Aab & $58.07 \mathrm{Aa}$ & $28.32 \mathrm{Abc}$ & $26.39 \mathrm{ABc}$ \\
\hline $0.4 \mathrm{~S}$ & $20.28 \mathrm{BCd}$ & $59.20 \mathrm{Aa}$ & $48.92 \mathrm{Aab}$ & $42.28 \mathrm{Abc}$ & $28.30 \mathrm{ABcd}$ \\
\hline $2 \mathrm{~S}$ & $11.21 \mathrm{CDd}$ & $60.41 \mathrm{Aa}$ & 47.82 Aab & $24.50 \mathrm{ABCcd}$ & $33.59 \mathrm{ABbc}$ \\
\hline \multirow[t]{2}{*}{$10 \mathrm{~S}$} & $1.64 \mathrm{Dbc}$ & $0.00 \mathrm{Bc}$ & $48.82 \mathrm{Aa}$ & $9.68 \mathrm{Cbc}$ & $16.96 \mathrm{Bb}$ \\
\hline & \multicolumn{5}{|c|}{ Purslane } \\
\hline 0 & $19.10 \mathrm{Aa}$ & $20.00 \mathrm{Aa}$ & $20.96 \mathrm{Aa}$ & $23.96 \mathrm{ABa}$ & $21.00 \mathrm{ABa}$ \\
\hline $0.4 \mathrm{R}$ & $19.07 \mathrm{Aa}$ & $9.39 \mathrm{BCb}$ & $11.60 \mathrm{Bb}$ & $11.96 \mathrm{CDb}$ & $7.85 \mathrm{Db}$ \\
\hline $2 \mathrm{R}$ & $18.80 \mathrm{ABab}$ & $15.39 \mathrm{ABb}$ & $24.21 \mathrm{Aa}$ & $17.57 \mathrm{Cb}$ & $16.10 \mathrm{BCb}$ \\
\hline $10 \mathrm{R}$ & $12.51 \mathrm{BCbc}$ & $17.53 \mathrm{Ab}$ & $26.96 \mathrm{Aa}$ & $24.89 \mathrm{Aa}$ & $11.63 \mathrm{CDc}$ \\
\hline $0.4 \mathrm{~S}$ & $7.62 \mathrm{CDc}$ & $21.50 \mathrm{Ab}$ & $23.39 \mathrm{Aab}$ & $27.35 \mathrm{Aa}$ & $23.28 \mathrm{Aab}$ \\
\hline $2 \mathrm{~S}$ & $2.44 \mathrm{Dd}$ & $20.57 \mathrm{Aab}$ & $24.82 \mathrm{Aa}$ & 18.21 BCbc & $13.57 \mathrm{CDc}$ \\
\hline \multirow[t]{2}{*}{$10 \mathrm{~S}$} & $2.50 \mathrm{Dc}$ & $4.88 \mathrm{Cbc}$ & $22.28 \mathrm{Aa}$ & $7.89 \mathrm{Dbc}$ & $9.86 \mathrm{CDb}$ \\
\hline & \multicolumn{5}{|c|}{ Pigweed } \\
\hline 0 & $29.53 \mathrm{Aab}$ & $27.75 \mathrm{Ab}$ & $37.75 \mathrm{Aa}$ & $31.85 \mathrm{Aab}$ & $31.43 \mathrm{Aab}$ \\
\hline $0.4 \mathrm{R}$ & $24.66 \mathrm{Aa}$ & $9.53 \mathrm{BCb}$ & $13.07 \mathrm{Bb}$ & $8.64 \mathrm{CDb}$ & $5.89 \mathrm{Bb}$ \\
\hline $2 \mathrm{R}$ & $23.32 \mathrm{ABb}$ & $24.32 \mathrm{Ab}$ & $38.74 \mathrm{Aab}$ & 32.26 Aab & $26.18 \mathrm{Ab}$ \\
\hline $10 \mathrm{R}$ & $13.00 \mathrm{BCc}$ & $18.64 \mathrm{ABbc}$ & $34.71 \mathrm{Aa}$ & $22.71 \mathrm{ABb}$ & 15.57 Bbc \\
\hline $0.4 \mathrm{~S}$ & $12.64 \mathrm{Cc}$ & $25.17 \mathrm{Ab}$ & $35.43 \mathrm{Aa}$ & $31.10 \mathrm{Aab}$ & $26.35 \mathrm{Aab}$ \\
\hline $2 \mathrm{~S}$ & $5.25 \mathrm{CDc}$ & $26.17 \mathrm{Aa}$ & $30.14 \mathrm{Aa}$ & $15.85 \mathrm{BCb}$ & 14.67 Bbc \\
\hline $10 \mathrm{~S}$ & $0.00 \mathrm{Dc}$ & $0.00 \mathrm{Cc}$ & $33.07 \mathrm{Aa}$ & $5.10 \mathrm{Dbc}$ & $10.60 \mathrm{Bb}$ \\
\hline
\end{tabular}

$\S$ Different concentrations $\left(0.4,2,10 \mathrm{~g} \mathrm{~L}^{-1}\right)$ of root $(\mathrm{R})$ or shoot $(\mathrm{S})$ extracts from sorghum-sudangrass plants harvested at 5 different stages $(10,20,30,40,50$ days after germination) were used to treat the seeds of four plant species (lettuce, tomato, purslane, and pigweed). For each species, plant length in the same column followed by the same upper case letter was not significantly different, while values in the same rows followed by the same lower case letter were not significantly different by the Tukey test $\mathrm{p} \leq 5 \%$.

$* \mathrm{R}=$ root extracts; $\mathrm{S}=$ shoot extracts. 
Table 4 - Lettuce, tomato, purslane, and pigweed shoot length after treatment with root and shoot aqueous extracts from sorghumsudangrass harvested at 5 growth stages ${ }^{\S}$

\begin{tabular}{|c|c|c|c|c|c|}
\hline \multirow{3}{*}{$\begin{array}{c}\text { Extract } \\
\text { concentration } \\
\left(\mathrm{g} \mathrm{L}^{-1}\right)\end{array}$} & \multicolumn{5}{|c|}{ Sorghum-sudangrass age (days after emergence) } \\
\hline & 10 & 20 & 30 & 40 & 50 \\
\hline & \multicolumn{5}{|c|}{ Shoots (mm) } \\
\hline & \multicolumn{5}{|c|}{ Lettuce } \\
\hline 0 & $15.67 \mathrm{Abc}$ & $11.78 \mathrm{Bc}$ & 14.10 Bbc & $18.07 \mathrm{Cb}$ & $24.03 \mathrm{BCb}$ \\
\hline $0.4 \mathrm{R}^{*}$ & $16.32 \mathrm{Ab}$ & $19.96 \mathrm{Aab}$ & $18.68 \mathrm{Aab}$ & $21.92 \mathrm{ABCa}$ & $22.53 \mathrm{BCa}$ \\
\hline $2 \mathrm{R}$ & $16.25 \mathrm{Abc}$ & $15.39 \mathrm{Bbc}$ & $12.68 \mathrm{Cc}$ & $18.92 \mathrm{Cab}$ & $21.96 \mathrm{BCab}$ \\
\hline $10 \mathrm{R}$ & $16.80 \mathrm{Abc}$ & $11.28 \mathrm{Bd}$ & $13.35 \mathrm{Bcd}$ & $20.96 \mathrm{BCa}$ & $19.96 \mathrm{Ca}$ \\
\hline $0.4 \mathrm{~S}$ & $13.64 \mathrm{ABb}$ & $12.93 \mathrm{Bb}$ & $15.46 \mathrm{ABCb}$ & $21.00 \mathrm{BCa}$ & $22.93 \mathrm{BCa}$ \\
\hline $2 \mathrm{~S}$ & $10.85 \mathrm{Bc}$ & $13.28 \mathrm{Bbc}$ & $17.35 \mathrm{ABb}$ & $24.28 \mathrm{ABa}$ & $26.39 \mathrm{ABa}$ \\
\hline \multirow[t]{2}{*}{$10 \mathrm{~S}$} & $0.00 \mathrm{Cd}$ & $6.68 \mathrm{Cc}$ & $17.00 \mathrm{ABbC}$ & $25.99 \mathrm{Aa}$ & $28.92 \mathrm{Aa}$ \\
\hline & \multicolumn{5}{|c|}{ Tomato } \\
\hline 0 & $24.89 \mathrm{ABa}$ & $23.50 \mathrm{Ba}$ & $28.15 \mathrm{Aa}$ & $23.34 \mathrm{Aa}$ & $21.12 \mathrm{Ca}$ \\
\hline $0.4 \mathrm{R}$ & $24.91 \mathrm{ABc}$ & $41.50 \mathrm{Aa}$ & $34.78 \mathrm{Aab}$ & $20.53 \mathrm{Ac}$ & $33.60 \mathrm{Ab}$ \\
\hline $2 \mathrm{R}$ & $24.25 \mathrm{Bb}$ & $26.75 \mathrm{Bab}$ & $32.02 \mathrm{Aa}$ & $24.17 \mathrm{Ab}$ & $23.42 \mathrm{BCb}$ \\
\hline $10 \mathrm{R}$ & $32.32 \mathrm{Aa}$ & $24.95 \mathrm{Bb}$ & $32.96 \mathrm{Aa}$ & $23.83 \mathrm{Ab}$ & 26.35 AabBC \\
\hline $0.4 \mathrm{~S}$ & $21.50 \mathrm{Bb}$ & $28.08 \mathrm{Bab}$ & $31.03 \mathrm{Aa}$ & $24.85 \mathrm{Aab}$ & $23.86 \mathrm{BCab}$ \\
\hline $2 \mathrm{~S}$ & $13.42 \mathrm{Cb}$ & $26.12 \mathrm{Ba}$ & $31.89 \mathrm{Aa}$ & $25.46 \mathrm{Aa}$ & $29.77 \mathrm{ABa}$ \\
\hline \multirow[t]{2}{*}{$10 \mathrm{~S}$} & $0.00 \mathrm{Da}$ & $0.00 \mathrm{Ca}$ & $33.28 \mathrm{Aa}$ & $24.64 \mathrm{Ab}$ & $30.03 \mathrm{ABab}$ \\
\hline & \multicolumn{5}{|c|}{ Purslane } \\
\hline 0 & $20.71 \mathrm{Aa}$ & $15.46 \mathrm{BCb}$ & $18.54 \mathrm{Cab}$ & $20.85 \mathrm{Da}$ & $20.28 \mathrm{Ba}$ \\
\hline $0.4 \mathrm{R}$ & $22.96 \mathrm{Aa}$ & $20.96 \mathrm{Aa}$ & $22.6 \mathrm{~A} \mathrm{BCa}$ & $22.46 \mathrm{CDa}$ & $23.99 \mathrm{ABa}$ \\
\hline $2 \mathrm{R}$ & $22.80 \mathrm{Aab}$ & $16.10 \mathrm{Bc}$ & $21.07 \mathrm{ABCb}$ & $25.92 \mathrm{ABa}$ & $22.71 \mathrm{Bab}$ \\
\hline $10 \mathrm{R}$ & $23.41 \mathrm{Aa}$ & $15.14 \mathrm{BCc}$ & $19.03 \mathrm{BCbc}$ & $22.39 \mathrm{CDab}$ & $23.75 \mathrm{Ba}$ \\
\hline $0.4 \mathrm{~S}$ & $15.69 \mathrm{Bc}$ & $13.93 \mathrm{BCc}$ & $20.39 \mathrm{ABCb}$ & $24.53 \mathrm{ABCab}$ & $24.93 \mathrm{ABa}$ \\
\hline $2 \mathrm{~S}$ & $10.69 \mathrm{Cd}$ & $15.46 \mathrm{BCc}$ & $23.28 \mathrm{ABb}$ & $28.53 \mathrm{Aa}$ & $23.28 \mathrm{Bb}$ \\
\hline \multirow[t]{2}{*}{$10 \mathrm{~S}$} & $3.28 \mathrm{Dc}$ & $10.78 \mathrm{Cb}$ & $24.86 \mathrm{Aa}$ & $24.46 \mathrm{ABCa}$ & $28.64 \mathrm{Aa}$ \\
\hline & \multicolumn{5}{|c|}{ Pigweed } \\
\hline 0 & $20.00 \mathrm{Ba}$ & $15.50 \mathrm{Ba}$ & $18.25 \mathrm{Ca}$ & $19.46 \mathrm{Ca}$ & $18.67 \mathrm{Ca}$ \\
\hline $0.4 \mathrm{R}$ & $22.30 \mathrm{Bb}$ & $35.93 \mathrm{Aa}$ & $32.17 \mathrm{Aab}$ & $29.96 \mathrm{Bb}$ & $23.21 \mathrm{BCa}$ \\
\hline $2 \mathrm{R}$ & $23.42 \mathrm{Ba}$ & $17.85 \mathrm{Bb}$ & 19.50 BCab & $23.11 \mathrm{Ca}$ & $19.43 \mathrm{Cab}$ \\
\hline $10 \mathrm{R}$ & $35.28 \mathrm{Aa}$ & $16.46 \mathrm{Bc}$ & $23.10 \mathrm{BCb}$ & $22.60 \mathrm{Cb}$ & $22.00 \mathrm{BCb}$ \\
\hline $0.4 \mathrm{~S}$ & $23.03 \mathrm{Bab}$ & $14.64 \mathrm{Bc}$ & 20.61 BCab & $23.85 \mathrm{Ca}$ & $18.53 \mathrm{Cbc}$ \\
\hline $2 \mathrm{~S}$ & $19.10 \mathrm{Bc}$ & $18.25 \mathrm{Bc}$ & $24.36 \mathrm{Bb}$ & $29.57 \mathrm{Ba}$ & $25.82 \mathrm{Bab}$ \\
\hline $10 \mathrm{~S}$ & $0.00 \mathrm{Cb}$ & $0.00 \mathrm{Cb}$ & $33.64 \mathrm{Aa}$ & $35.75 \mathrm{Aa}$ & $32.35 \mathrm{Aa}$ \\
\hline
\end{tabular}

$\S$ Different concentrations $\left(0.4,2,10 \mathrm{~g} \mathrm{~L}^{-1}\right)$ of $\operatorname{root}(\mathrm{R})$ or shoot $(\mathrm{S})$ extracts from sorghum-sudangrass plants harvested at 5 different stages $(10,20,30,40,50$ days after germination) were used to treat the seeds of four plant species (lettuce, tomato, purslane, and pigweed). For each species, plant length in the same column followed by the same upper case letter was not significantly different, while values in the same rows followed by the same lower case letter were not significantly different by the Tukey test pd" $5 \%$.

$* \mathrm{R}=$ root extracts; $\mathrm{S}=$ shoot extracts. 


\section{LITERATURE CITED}

AN, M. et al. Mathematical modeling of allelopathy: II. The dynamics of allelochemicals from living plants in the environment. Ecol. Modeling, v. 116, n.1, p. 53-66, 2003.

BAIS, H. P. et al. The role of root exudates in rhizosphere and other organisms. An. Rev. Plant Biol., v. 57, p. 233-66, 2006

BÀRBERI, P.; MAZZONCINI, M. Changes in weed community composition as influenced by cover crop and management system in continuous corn. Weed Sci., v. 49, n. 4, p. 491-499, 2001.

BARBOSA, T. M. L. et al. Caracterização química e efeitos alelopáticos de exsudatos radiculares de plântulas de sorgo sobre alface. Planta Daninha, v. 16, n. 2, p. 153-162, 1998.

BEN-HAMMOUDA, M. et al. A chemical basis for differential allelopathic potential of sorghum hybrids on wheat. J. Chem. Ecol., v. 21, n. 6, p. 775-786, 1995.

BLYTH, C. R. Aproximate binomial confidence limits. J. Am. Stat., v. 81, n. 385, p. 843-855, 1996.

CORREIA, N. M.; CENTURION, M. A. P. C.; ALVES, P. L. C. A. Influência de extratos aquosos de sorgo sobre a germinação e desenvolvimento de plântulas de soja. Ci. Rural, v. 35, n. 3, p. 498-503, 2005a.

CORREIA, N. M.; SOUZA, I. F.; KLINK, U. P. Palha de sorgo associada ao herbicida imazamox no controle de plantas daninhas na cultura da soja em sucessão. Planta Daninha, v.23, n. 3, p. 483-489, 2005b

CZARNOTA, M. A.et al. Mode of action, localization of production, chemical nature, and activity of sorgoleone: a potent PSII inhibitor in Sorghum spp. root exudates. Weed Technol., v. 15, n. 4, p. 813-825, 2001.

DAYAN, F. E. Factors modulating the levels of the allelochemical sorgoleone in Sorghum bicolor. Planta, v. 224, n. 2, p. 339-346, 2006.

EVERITT, J. H.; ALANIZ, M. A.; LEE, J. B. Seed germination characteristics of Kochia scoparia. J. Range Manag., v. 36, n. 5, p. 646-648, 1983.

FERREIRA, D. F. Análises estatísticas por meio do Sisvar para Windows versão 4.0. In: REUNIÃO ANUAL DA REGIÃO BRASILEIRA DA SOCIEDADE INTERNACIONAL DE BIOMETRIA, 45., São Carlos, 2000. Proceedings... São Carlos, Universidade Federal de São Carlos, 2000. p. 255-258.
FORNEY, D. R.; FOY, C. L. Phytotoxicity of products from rhizospheres of a sorghum-sudangrass hybrid (Sorghum bicolor x Sorghum sudanense). Weed Sci., v. 33, n. 5, p. $597-604,1985$.

GONZALEZ, V. M. et al. Inhibition of a photosystem II electron transfer reaction by the natural product sorgoleone. J. Agric. Food Chem., v. 45, n. 4, p. 1415-1421, 1997.

HEJ, A. M.; KOSTER, K. L. The allelochemical sorgoleone inhibits root H+-ATPase and water uptake. J. Chem. Ecol., v. 30, n. 11, p. 2181-2191, 2004.

LEEMIS, L. M.; TRIVEDI, K. S. A comparison of approximate interval estimators for the Bernoulli parameter. Am. Stat., v. 50, n. 1, p. 63-68, 1996.

MCGUIRE, A. Cover crops for the Columbia basin. Washington: Cooperative Extension Washington State University, 2003. 2 p. (EB1950E).

MCKINNEY, D. E. et al. A preliminary study of dual use of cover crops: sorghum-sudangrass as both hay and summer cover crop for no-till organic cabbage. In: SOUTHERN CONSERVATION TILLAGE CONFERENCE FOR SUSTAINABLE AGRICULTURE, 26., 2004, Raleigh. Proceedings... Raleigh: 2004. 10p.

MELLO, R. et al. Análise produtiva e qualitativa de um híbrido de sorgo interespecífico submetido a dois cortes. R. Bras. Milho Sorgo, v. 2, n. 1, p. 20-33, 2003.

NETZLEY, D. H.; BUTLER, L. G. Roots of sorghum exude hydrophobic droplets containing biologically active components. Crop Sci., v. 26, n. 4, p. 775-780, 1986.

NICOLLIER, G. F.; POPE, D. F; THOMPSON, A. C. Biological activity of dhurrin and other compounds from johnson grass (Sorghum halepense). J. Agric. Food Chem., v. 31, n. 4 , p. $748-751,1983$.

OLIBONE, D. et al. Crescimento inicial da soja sob efeito de resíduos de sorgo. Planta Daninha, v. 24, n. 2, p. 255-261, 2006.

PRATT, J. W. A normal approximation for binomial, F, beta, and other common, related tail probabilities, II. J. Am. Station., v. 63, n. 324, p. 1457-1483, 1968.

RAUPP, A. A. A.; BRANCÃO, N. Resultados de área de validação de tecnologia com sorgo de corte/pastejo submetido a pastejo rotativo 1999/2000. In: REUNIÃO TÉCNICA DO MILHO, 45.; REUNIÃO TÉCNICA DO SORGO, 28., 2000, Pelotas. Anais... Pelotas: Embrapa Clima Temperado, 2000. p. 673. 
ROTH, C. M.; SHROYER, P.; PAULSEN, G. M. Allelopathy of sorghum on wheat under several tillage systems. Agron. J., v. 92, n. 5, p. 855-860, 2000.

SENE, M.; GALLET, C.; DORÉ, T. Phenolic compounds in a Sahelian sorghum (Sorghum bicolor) genotype (ce(14566)) and associated soils. J. Chem. Ecol., v. 27, n. 1, p. 8192,2001

TOMICH, T. R. et al. Potencial forrageiro de híbridos de sorgo com capim-sudão. Arq. Bras. Med.Veter. Zoot., v. 6, n. 2 , p. $258-263,2004$

TREZZI, M. M.; VIDAL, R. A. Potencial de utilização de cobertura vegetal de sorgo e milheto na supressão de plantas daninhas em condição de campo: II - efeitos da cobertura morta. Planta Daninha, v. 22, n. 1, 1-10, 2004.

VIDAL, R. A.; TREZZI, M. M. Potencial da utilização de coberturas vegetais de sorgo e milheto na supressão de plantas daninhas em condição de campo: I - Plantas em desenvolvimento vegetativo. Planta Daninha, v. 22, n. 2 , p. 217-223, 2004.
WESTON, L.A. Utilization of allelopathy for weed management in agroecosystems. Agron. J., v. 88, p.860-866, 1996.

WESTON, L.A.; HARMON, R.; MUELLER, S. Allelopathic potential of sorghum-sudangrass hybrid (sudex). J. Chem. Ecol., v. 15, n. 6, p. 1855-1865, 1989.

WOODHEAD, S. Environmental and biotic factors affecting the phenolic content of different cultivars of Sorghum bicolor. J. Chem. Ecol., v. 7, n. 7, p. 1035-1047, 1981.

XUAN, T. D. et al. Methods to determine allelopathic potential of crop plants for weed control. Allelopathy J., v. 13, n. 2 , p. 149-164, 2004.

ZAGO, C. P. Utilização do sorgo na alimentação de ruminantes. In: EMPRESA BRASLEIRA DE PESQUISA AGROPECUÁRIA - Centro Nacional de Pesquisa de Milho e Sorgo. Manejo cultural do sorgo para forragem. Sete Lagoas: 1997. p. 9-26. (Embrapa-CNPMS. Circular Técnica, 17) 